\section{Q de Cohen: Comparación de Correlaciones entre Muestras Independientes en base a Urzúa et al}

\section{Comparing Independent Samples Correlations: suggestions based of Urzúa et al}

\section{Sr. Editor}

En investigación es común realizar estudios de relación entre dos variables; sin embargo, es poco habitual la práctica de comparar las correlaciones entre el género, edad, estado civil u otra variable sociodemográfica, con el fin de estimar la posible influencia de los grupos en la correlación general. Este es el caso del importante trabajo de Urzúa, Heredia y Caqueo-Urízar ${ }^{1}$, quienes analizan la relación entre el estrés por aculturación y la presencia de sintomatología relacionada a problemas de salud mental en inmigrantes colombianos y peruanos radicados en Chile. La lectura de este estudio nos lleva a preguntarnos ¿las correlaciones entre las puntuaciones del estrés por aculturación y los síntomas asociados a la salud mental son diferentes entre inmigrantes colombianos y peruanos, más aun sabiendo que estas personas provienen de contextos culturales diferentes?. La ausencia de información en el artículo de Urzúa et al no permite realizar los cálculos necesarios para responder concretamente a esta pregunta, pero da pie a sugerir el empleo de la $q$ de Cohen como un aporte metodológico que permita valorar si las diferencias entre las correlaciones de los grupos son importantes.

La $q$ es uno de los tantos coeficientes que planteó Cohen ${ }^{2}$, para el cálculo del tamaño del efecto, empleada específicamente en la comparación de coeficientes de correlación en muestras independientes. Consiste en trasformar los coeficientes de correlación de Pearson en coeficientes $\mathrm{Z}$ de Fisher, mediante la siguiente formula ${ }^{3}$ :

$$
\frac{1}{2} \ln \left[\frac{1+r}{1-r}\right]
$$

Donde ln, es logaritmo y $r$ es la correlación. La ventaja de la transformación de $r$ a $z$ de Fisher es la aproximación a una distribución normal ${ }^{4}$. Recientemente se ha demostrado que esta trasformación carece de sesgo, aunque puede estar afectada por la normalidad bivaria$\mathrm{da}^{5}$. Si las distribuciones de los residuos son normales, la distribución de la $\mathrm{Z}$ de Fisher tiende a la normalidad ${ }^{6}$. Del mismo modo, se puede calcular el error estándar mediante la siguiente formula ${ }^{4}$ :

$$
e e=\frac{1}{\sqrt{n-3}}
$$

Una vez que se convierta cada coeficiente de correlación de Pearson a Z de Fisher. La Q de cohen quedará definida como la diferencia entre las transformaciones $\mathrm{Zs}^{2}$ :

$$
q_{\text {cohen }}=z_{r 1}-z_{r 2}
$$

Dónde: $Z_{\mathrm{n} 1}$ y $Z_{\mathrm{n} 2}$ son las transformaciones en $\mathrm{Z}$ de Fisher para cada una de las correlaciones. Adicionalmente, en base a lo señalado por Loftus \& Loftus ${ }^{7}$, se puede establecer intervalos de confianza definidos como, la $q$ de Cohen más/menos el coeficiente de confianza multiplicado por el error estándar, siendo su expresión matemática la siguiente:

$$
I C=q_{\text {cohen }} \pm z_{1-(\alpha / 2)} \sqrt{\frac{1}{n-3}}
$$


Dónde: IC es el intervalo de confianza; $Z_{1-(\alpha / 2)}$ es 1,$96 ; \mathrm{n}$ es la cantidad de personas. El cálculo de los intervalos de confianza permite tener una información acerca de la magnitud y la precisión de la relación ${ }^{8}$.

Para efectos de ejemplificar su uso, partiremos de un caso supuesto. En un estudio, que tiene como objetivo establecer si la relación entre las puntuaciones de bienestar y la imagen corporal de 79 adolescentes de 12 a 18 años son diferentes entre aquellos diagnosticados con trastornos de conductas alimentarias y los no diagnosticados, se obtuvo una correlación de 0,56 entre las variables para el grupo de pacientes con trastornos de conducta alimentaria $\left(\mathrm{n}_{1}=40\right)$ y una correlación de 0,47 para el grupo sin trastorno $\left(\mathrm{n}_{2}=39\right)$. En primer lugar, se realiza la transformación de los coeficientes de correlación como se ilustra a continuación, tanto en el caso de los hombres como el de mujeres:

$$
\begin{aligned}
& \frac{1}{2} \ln \left[\frac{1+0,56}{1-0,56}\right]=0,63 \quad \text { (trastornos de cond } \\
& \frac{1}{2} \ln \left[\frac{1+0,47}{1-0,47}\right]=0,51 \quad \text { (sin trastorno) }
\end{aligned}
$$

Finalmente, la $q$ de Cohen sería la diferencia entre los dos valores transformados: $q_{\text {cohen }}=0,63-0,51=0,123$, mientras que el cálculo de los intervalos de confianza, arroja los siguientes resultados:

$$
\begin{aligned}
& l i=0,123+1,96 \sqrt{\frac{1}{79-3}=}-0,102 \\
& l s=0,123-1,96 \sqrt{\frac{1}{79-3}}=0,348
\end{aligned}
$$

Una vez obtenido el valor de la $q$, es pertinente utilizar las categorías interpretativas planteadas por Cohen $^{2}$, en donde valores <0,10: sin efecto; 0,10 a 0,30: efecto pequeño; 0,31 a 0,50 : efecto moderado; $>0,51$ : efecto grande. En nuestro ejemplo, un valor de la $q$ de Cohen de 0,12 indicaría que la proporción de la diferencia entre la correlación de bienestar e imagen corporal según diagnóstico o no de trastornos de la conducta alimentaria es pequeña; además, de acuerdo a los intervalos de confianza, el valor de la $q$ de Cohen no fue estadísticamente significativo, dado que el intervalo incluye al cero.

Cabe mencionar que en el caso de una correlación de Spearman, no existe argumento para el uso de la $q$ de Cohen; sin embargo, podría utilizarse la transformación de Rupinski y Dunlap ${ }^{9}$ para convertir la Rho de Spearman en $r$ de Pearson, cuya expresión matemática es:

$$
r=2 \operatorname{sen}\left(r_{s} \frac{\pi}{6}\right)
$$

Dónde: sen es el seno; $r$ s es la Rho de Spearman, $\varpi$ es el valor 3.1416. Finalmente, una vez realizada transformada la Rho a r, se debe proceder a convertirlo a Z de Fisher. Rupinski y Dunlap (1996) en su estudio de simulación encontraron que las transformaciones de rho de Spearman a $r$ de Pearson presentan superiores errores estándar al $r$ de Pearson, aunque dicho incremento es insignificante $(-0,005)$ a nivel práctico. Por tanto, brinda correlaciones aceptables para estudios de metaanálisis.

En el caso del estudio de Urzúa et al, el empleo de la $q$ de Cohen permitiría determinar si las correlaciones entre las variables pueden ser diferentes en función de las diferencias idiosincráticas de los inmigrantes peruanos y colombianos. Si bien en la discusión se hace referencia a una posible influencia de estas diferencias, no se hace mención alguna sobre datos empíricos que respalden esta aseveración, recomendándose así, procedimientos metodológicos que permiten obtener información complementaria respecto a la comparación de las correlaciones entre grupos independientes, obteniendo así una mejor evidencia para la formulación de conclusiones. Con el fin de agilizarse los cálculos, se ha elaborado una hoja de Excel para realizar las conversiones de forma automática, la cual puede ser solicitada sin costo a alguno a los autores.

José Luis Ventura-León ${ }^{a}$, Tomás Caycho ${ }^{a}$ Universidad Privada del Norte, Lima, Perú. ${ }^{a}$ Magister en Psicología.

\section{Referencias}

1. Urzúa A, Heredia O, Caqueo-Urízar A. Salud mental y estrés por aculturación en inmigrantes sudamericanos en el norte de Chile. Rev Med Chile 2016; 144 (5): 563-70.

2. Cohen J. Statistical Power Analysis for the Behavioral Sciences. 2nd ed. New York: Erlbaum, Hillsdale; 1988.

3. Fisher RA. Frequency distribution of the values of the correlation coefficient in samples from an indefinitely large population. Biometrika 1915; 10: 507-21.

4. Fisher RA. On the "probable error" of a coefficient of correlation deduced from a small sample. Metron 1921; 1: 3-32.

5. Zimmerman DW, Zumbo BD, Williams RH. Bias estimation and hypothesis testing of correlation. Psicológica 2003; 24: 133-58.

6. Sánchez-Bruno A, Borges del Rosal, A. Estudio de la distribución muestral de la transformación $\mathrm{Z}$ de Fisher. Metodología de las Ciencias del Comportamiento 2004; 1: 543-8.

7. Loftus GR, Loftus EF. Essence of statistics, 2nd ed. New York, Random House, 1988.

8. Zou GY. Toward using confidence intervals to compare correlations. Psychol Methods 2007; 12: 399-413.

9. Rupinski MT, Dunlap WP. Approximating Pearson product-poment correlations from Kendall's Tau and Spearman's Rho. Educ Psychol Meas 1996; 56 (3): 419-29. 